\title{
A genetic variant in osteoprotegerin is associated with progression of joint destruction in rheumatoid arthritis
}

Rachel Knevel ${ }^{1 *}$, Diederik PC de Rooy ${ }^{1}$, Tore Saxne ${ }^{2}$, Elisabet Lindqvist ${ }^{2}$, Martha K Leijsma ${ }^{3}$, Nina A Daha ${ }^{1}$, Bobby PC Koeleman ${ }^{4}$, Roula Tsonaka ${ }^{5}$, Jeanine J Houwing-Duistermaat ${ }^{5}$, Joris JM Schonkeren ${ }^{1}$, Rene EM Toes ${ }^{1}$, Tom WJ Huizinga ${ }^{1}$, Elisabeth Brouwer ${ }^{3}$, Anthony G Wilson ${ }^{6}$ and Annette HM van der Helm-van Mil ${ }^{1}$

\begin{abstract}
Introduction: Progression of joint destruction in rheumatoid arthritis (RA) is partly heritably; 45 to $58 \%$ of the variance in joint destruction is estimated to be explained by genetic factors. The binding of RANKL (Receptor Activator for Nuclear Factor K B Ligand) to RANK results in the activation of TRAF6 (tumor necrosis factor (TNF) receptor associated factor-6), and osteoclast formation ultimately leading to enhanced bone resorption. This bone resorption is inhibited by osteoprotegerin (OPG) which prevents RANKL-RANK interactions. The OPG/RANK/RANKL/ TRAF6 pathway plays an important role in bone remodeling. Therefore, we investigated whether genetic variants in $O P G, R A N K$, RANKL and TRAF6 are associated with the rate of joint destruction in RA.
\end{abstract}

Methods: 1,418 patients with 4,885 X-rays of hands and feet derived from four independent data-sets were studied. In each data-set the relative increase of the progression rate per year in the presence of a genotype was assessed. First, explorative analyses were performed on 600 RA-patients from Leiden. 109 SNPs, tagging OPG, RANK, RANKL and TRAF6, were tested. Single nucleotide polymorphisms (SNPs) significantly associated in phase-1 were genotyped in data-sets from Groningen (Netherlands), Sheffield (United Kingdom) and Lund (Switzerland). Data were summarized in an inverse weighted variance meta-analysis. Bonferonni correction for multiple testing was applied.

Results: We found that 33 SNPs were significantly associated with the rate of joint destruction in phase-1. In phase-2, six SNPs in OPG and four SNPs in RANK were associated with progression of joint destruction with $P$-value $<0.05$. In the meta-analyses of all four data-sets, RA-patients with the minor allele of OPG-rs 1485305 expressed higher rates of joint destruction compared to patients without these risk variants $\left(P=2.35 \times 10^{-4}\right)$. This variant was also significant after Bonferroni correction.

Conclusions: These results indicate that a genetic variant in $O P G$ is associated with a more severe rate of joint destruction in RA.

\section{Introduction}

Rheumatoid Arthritis (RA) is an autoimmune disorder that affects $0.5-1 \%$ of the population and is associated with significant morbidity, disability and costs for society. Radiographic joint destruction reflects the cumulative burden of inflammation and is conceived as an objective measure of RA severity [1]. The degree of joint destruction varies significantly between patients. The processes behind

\footnotetext{
* Correspondence: r.knevel@lumc.nl

${ }^{1}$ Department of Rheumatology, Leiden University Medical Center, Leiden, the Netherlands

Full list of author information is available at the end of the article
}

this difference are incompletely understood. Inflammatory markers and auto-antibodies are known risk factors for joint destruction but explain approximately $30 \%$ of the total variance in joint destruction [2]. A twin study suggested that genetic factors influence the severity of joint destruction in RA and a recent study in the Icelandic RApopulation estimated the heritability of the rate of joint destruction around $45-58 \%[3,4]$. Hence, to increase the understanding of progression mediating disease processes, it seems valuable to study genetic variants that could predispose to joint destruction in RA.

\section{Biomed Central}


The balance between osteoblast and osteoclast activity is crucial for healthy bone and is disturbed in systemic or local conditions that affect the skeleton such as osteoporosis or RA. Figure 1 schematically depicts the OPG/ RANK/RANKL/TRAF6 pathway which mediates osteoclast related bone loss. RANKL (Receptor Activator for Nuclear Factor $\mathrm{k}$ B Ligand) is expressed and released by osteoblasts and activated T lymphocytes [5]. RANKL promotes osteoclast formation and perpetuate their function and survival through binding of RANK (Receptor Activator of Nuclear Factor $\mathrm{k}$ B). Subsequently, the signal of RANK is mediated by TRAF6, a member of the TNF receptor associated factor (TRAF) protein family, which functions as a signal transducer in the NF $\kappa \beta$ family [6]. The process of osteoclast formation and bone resorption is also regulated by OPG (osteoprotegerin), which is secreted by osteoblasts. By binding of OPG to RANKL, activation of the RANK receptor is inhibited.

The net bone loss in RA suggests that there is an imbalance in the OPG-RANKL axis favoring bone resorption and resulting in erosions [6-8]. Recent studies showed that in RA RANKL is, amongst others, expressed in cultured synovial fibroblasts, chondrocytes and by CD4+ and CD8+ $\mathrm{T}$ lymphocytes [5,9-11]. In addition, the ratio of OPG/ RANKL serum levels is associated with joint destruction in RA [12]. Furthermore, several studies have observed an association of genetic variants in OPG, RANK or RANKL with bone mineral density and osteoporosis [13-16]. Together, these data led us to hypothesize that genetic variants in OPG, RANK, RANKL and TRAF6 are associated with the severity of joint destruction in RA. We tested this hypothesis using four data-sets of European RA-patients with longitudinal radiological data on joint destruction. All data-sets included patients that were diagnosed in a period when treatment strategies were less aggressive and disease activity was less controlled than today. These conservative treatment strategies made these data-sets suitable for the present study as the natural course of disease was less inhibited.

\section{Methods \\ Study population}

Four data-sets consisting of adult European RA-patients were studied. RA was defined according to the 1987 ACR criteria in all data-sets except for the Lund data-set where the 1958 ACR-criteria were used. X-rays of both hands and feet were available for all patients (Table 1). All patients gave their informed consent and approval was obtained from the local Ethical Committee of each hospital (METC Leiden, EPN Lund, METC Groningen, COREC Sheffield).

\section{Leiden-early arthritis clinic cohort (Leiden-EAC)}

This cohort contained 600 early RA-patients from the western part of the Netherlands, who were included between 1993 and 2006 [2]. Arthritis patients were included at the first visit at the outpatient clinic and yearly followed. Blood samples were collected at baseline. DNA was extracted en preserved for later usage. X-rays were taken at baseline and on yearly follow-up visits during 7years. In total, 2,846 sets of hands and feet X-rays were available. All X-rays were chronologically scored by one experienced reader who was unaware of genetic or clinical data using the Sharp-van der Heijde scoring method (SHS) on hands and feet [17]. This method quantifies both joint-space-narrowing, a feature of cartilage loss, and bony erosions. It is a semi-quantitative method, the maximal total scores is 448.499 randomly selected X-rays

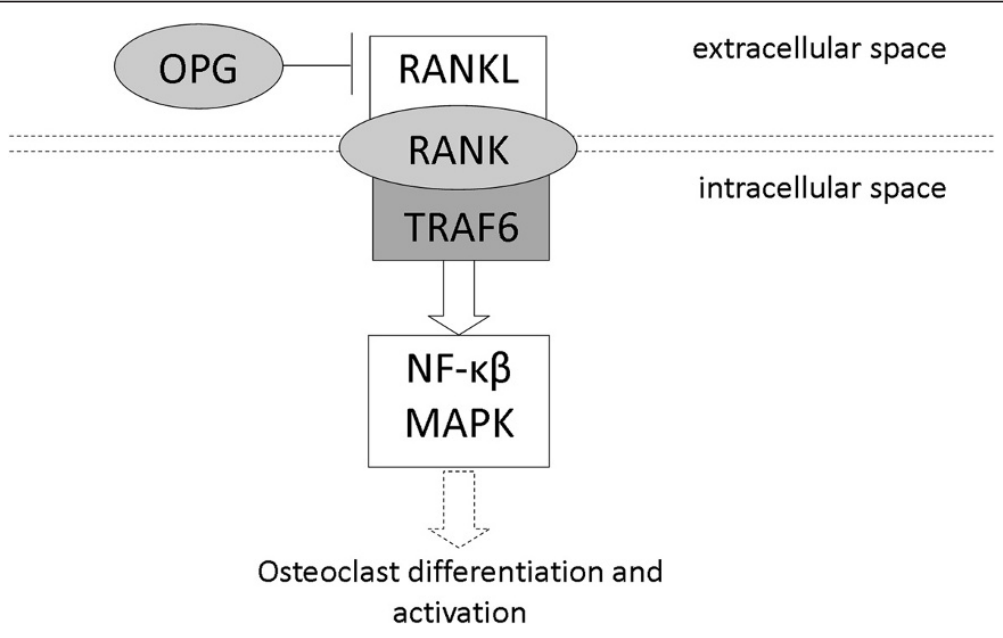

Figure 1 Schematic presentation of the OPG/RANK/RANKL/TRAF6 pathway in osteoclasts. The RANK signaling cascade is initiated upon the binding of RANKL to the extracellular domain of RANK which panes the signal along to TRAF6. The activation of TRAF6 initiates pathways leading to the activation of several transcription factors (among which NFK 3 and MAP kinase mediators), which contribute to osteoclast differentiation, activation and survival. OPG is able to prevent the interaction between RANKL and RANK. 
Table 1 Characteristics for each data-set

\begin{tabular}{|c|c|c|c|c|}
\hline Cohort & $\begin{array}{l}\text { Leiden-EAC } \\
(n=600)\end{array}$ & $\begin{array}{l}\text { Groningen } \\
(\mathrm{n}=275)\end{array}$ & $\begin{array}{l}\text { Sheffield } \\
(\mathrm{n}=391)\end{array}$ & $\begin{array}{l}\text { Lund } \\
(n=147)\end{array}$ \\
\hline Year of diagnosis & 1993-2006 & $1945-2001$ & $1938-2003$ & 1985-1990 \\
\hline Follow-up years* & 7 years & 14 years & Not applicable* & 5 years \\
\hline Total no. of X-ray sets & 2,846 & 862 & 391 & 781 \\
\hline Method of scoring & SHS & SHS & Larsen & Larsen \\
\hline Female $\mathrm{n}(\%)$ & $412(69)$ & $194(71)$ & $290(73)$ & $98(67)$ \\
\hline Age at diagnosis, mean \pm SD & $56 \pm 16$ & $49 \pm 13$ & $46 \pm 13$ & $51 \pm 12$ \\
\hline $\mathrm{ACPA}+\mathrm{n}(\%)$ & $323(55)$ & $160(80)$ & $302(79)$ & $114(80)$ \\
\hline Rheumatoid factor $\mathbf{n}(\%)$ & $343(59)$ & $258(94)$ & $N / A^{*}$ & $115(81)$ \\
\hline
\end{tabular}

SHS Sharp-van der Heijde score.

*Data of Leiden-EAC, Groningen and Lund were from baseline onwards during respectively 7, 14 and 5 years of follow-up. The data of Sheffield were collected once during the disease period, the mean disease duration was 15 years (range 3-65 years).

N/A not available.

were scored twice. The correlation coefficient (ICC) within the reader was 0.91 . The treatment of these patients could be divided into three treatment periods. Patients included in 1993-1995 were initially treated with NSAIDs, patients included in 1996-1998 were initially treated with chloroquine or sulphasalazine and patients included after 1999 were promptly treated with methotrexate or sulphasalazine.

\section{Groningen}

The second set of data involved 275 RA-patients from the Northern part of the Netherlands that were diagnosed in 1945-2001. The follow-up duration after diagnosis was limited to 14-years. The mean number of X-ray sets (hands and feet) per patient was 3.1 (with a maximum of eight Xrays per patient). The total number of sets of X-rays was 862. The X-rays were scored chronologically by one of two readers using SHS. ICCs within readers were $>0.90$ and between readers 0.96 . The development of joint destruction was significantly different for patients included after 1990 compared to patients included before 1990. This observation is in line with the introduction of early initiation of treatment with Disease Modifying Anti-Rheumatic Drugs (DMARDs) after 1990.

\section{Sheffield}

The third set of patients concerned 391 RA-patients from the area of Sheffield, UK. RA-patients with X-rays available were recruited from the Rheumatology department of the Royal Hallamshire Hospital in Sheffield between 1999 and 2006 [17]. RA-patients were assessed once during their disease course. The mean $( \pm S D)$ disease duration at assessment was $15 \pm 11$ years (range 365 years). X-rays of hands and feet were scored by one reader using a modification to Larsen's score [18]. This method quantifies the severity of bony erosions and joint space narrowing in one score, both elements are not scored separately. Ten percent of films were scored twice to quantify the intra-observer variation by a weighted kappa score which was 0.83 [19].

\section{Lund}

This cohort concerned 183 Swedish early RA-patients that were prospectively followed yearly during 5-years, of which 147 had X-rays and DNA available [18,20]. Patients were recruited from primary care units in the area of Lund during 1985-1989. X-rays of hands and feet were taken at study start and annually for 5-years, resulting in a total of 781 sets of X-rays. X-rays were scored chronologically according to Larsen by one of two readers [21]. The ICC between the readers determined on $105 \mathrm{X}$-rays was 0.94 . In the inclusion period, immediate DMARD-therapy was not common and at 5-years follow-up still a substantial proportion of the patients were not treated with a DMARD. The most commonly used DMARDs were chloroquine, Dpenicillamin, sodium aurothiomalate and auranofin [22].

\section{SNP selection and genotyping}

The region of OPG, RANK, RANKL and TRAF6 plus the haplotype blocks up- and downstream these genes were tagged by the algorithm of HaploView [23]. One SNP in OPG, two in RANK and one in TRAF6 were known to be amino acid changing SNPs; respectively rs2073618, rs1805034, rs8092336 and rs3740958. Eight SNPs were associated with bone mineral density in the hip or spine in previous studies; OPG rs6993813 [13], rs646980 [13], rs4355801 [14] and rs2073618 [15]; RANK rs3018362 [13] and rs884205 [16]; RANKL rs9594738 [13] and rs9594759 [13]. All these SNPs were forced to include. Pairwise tagging SNPs were selected from the CEPH/ CEU hapmap data-set (phase II, release 21, NCBI build 35) using haploview software (MAF $>0.05$, pairwise $\mathrm{r} 2>0.8)$. In total 109 SNPs captured OPG (34), RANK (54), RANKL (21) and TRAF6 (17). Multiplex 
SNPs arrays were designed using Illumina Golden Gate platform, according to the protocols recommended by the manufacturer [Illumina, San Diego, CA]. Three SNPs could not be designed; rs10505348, rs7239667 and rs9951012. Proxies were sought and found for rs10505348: rs4355801 $\left(r^{2}=0.80\right)$, for the other two SNPs no good proxy existed.

Software supplied by Illumina was used to automatically identify the genotypes. Each 96-wells plate consisted of 1 positive and 1 negative control, which were all indeed tested positive and negative. Clusters were evaluated and all doubtful calls were checked; after manually evaluating the spectra of each cluster, the genotypes were accepted, recalled or rejected. At least $12 \%$ of the genotypes were assessed in duplicate, with an error rate of $<2.5 \%$ for all SNPs. Genetic sex was checked with the reported sex in the database. SNPs were selected if the success rate were $\geq 95 \%$ and the Hardy-Weinberg equilibrium (HwE) p-value $>0.001$.

SNPs that had a clear and significant association with joint destruction in the first cohort were selected to be genotyped in the other three data-sets. The SNPs were genotyped as a part of multiplex SNPs arrays designed with Sequenom iPLEX, according to the protocols recommended by the manufacturer [Sequenom, San Diego, California]. Software supplied by the same manufacturer was used to automatically identify the genotypes. Two SNPs could not be designed but full proxies $\left(\mathrm{r}^{2}=1.0\right)$ were typed instead (rs17666267:rs9959310 and rs1564861: rs3134057). Each iPLEX consisted of at least 9 positive and 9 negative controls, which were indeed tested positive and negative. All doubtful calls were checked manually, DNA samples with $>30 \%$ failed SNPs were excluded from analysis $(\mathrm{n}=31)$. At least $5 \%$ of the genotypes were assessed in duplicate, with an error rate of $<1 \%$. SNPs were selected if the success rate were $\geq 95 \%$ and HwE p-value $>0.001$.

\section{Statistical analysis}

Associations between genotypes and radiographic joint destruction were analyzed. Two phases were carried out. An overview of the SNP selection process is provided in Figure 2. First, an explorative analysis was performed in the Leiden-EAC. In this data-set the tagged SNPs were tested in two ways; additively and recessively. Since phase-1 was an explorative phase no correction for multiple testing was applied yet and SNPs with a $\mathrm{p}$-value $<0.05$ were studied in phase- 2 .

Significant SNPs from phase-1 were preceded in phase- 2 if they had a high enough minor allele frequency and if the effect was independent of other SNPs (see Additional file 1: Table S1). In phase-2 SNPs were analyzed in three independent data-sets and meta-analyses of all four data-sets. In the present study the power to detect genetic effects is a function of the number of patients and the number of measurements per patient

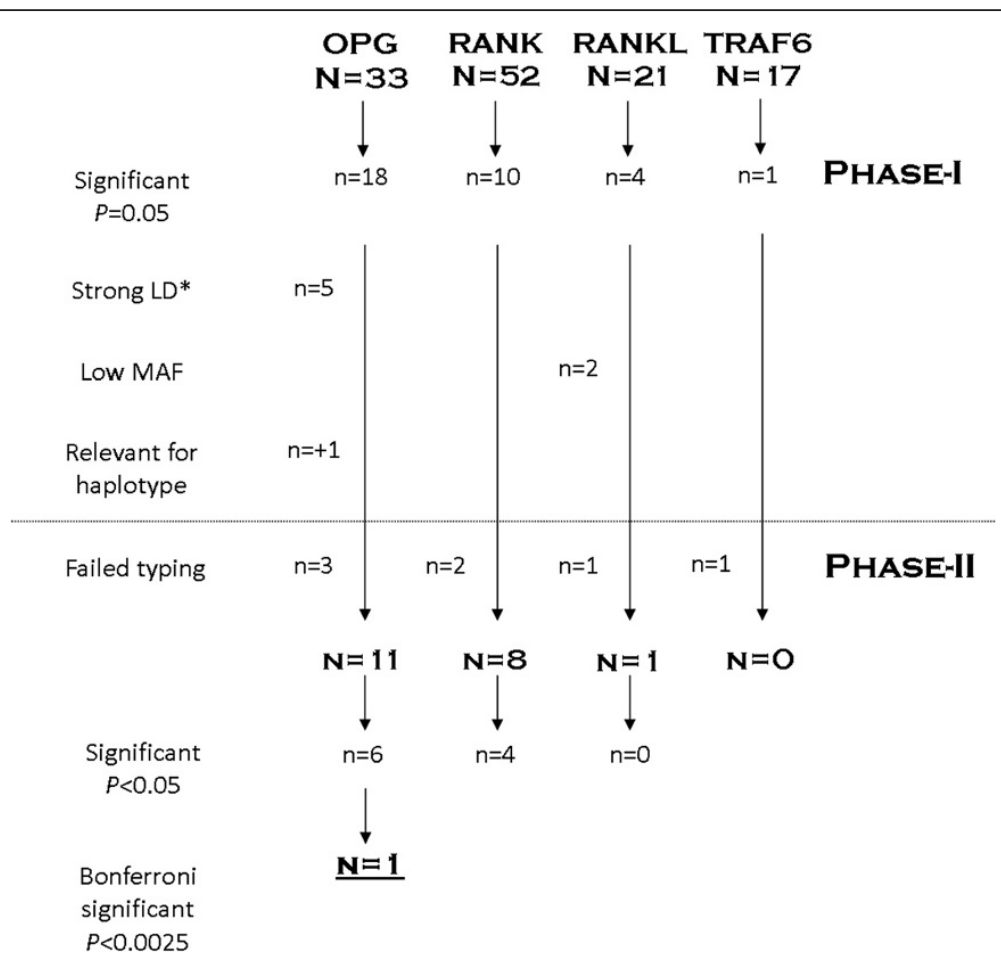

Figure 2 Schematic depiction of the SNPs selection process. *LD is linkage disequilibrium, some SNPs were in strong correlation in the dataset of phase-1 as calculated with $\mathrm{R}^{2}$ in haploview, or were part of a haplotype were other SNPs caused the significant association. 
studied $[24,25]$. All three data-sets studied to verify the results of phase- 1 contained (individually and combined) less X-rays than the initial data-set. Consequently, the power to replicate findings in each data-set individually as well as in the three replication data-sets together was expected to be limited due to a lower number of X-rays than in the discovery data-set. Therefore it was decided to test the SNPs in each data-set separately taking advantage of the specific data-set characteristics and to subsequently perform a meta-analysis on the results to determine the association of the SNPs with the rate of joint destruction. An inverse variance weighting metaanalysis testing for fixed effect $[26,27]$ was performed in Stata, version 10.1. In phase-2 analyses were performed either additively or recessively depending of the findings of phase-1. To provide insight into the heterogeneity of the cohorts the $I^{2}$ for heterogeneity was calculated.The choice for a fixed effect model was guided by the argument of Lebrec et al. [27] that the heterogeneity is of less importance in genetic studies when the search for a significant association in the first studies is of more importance than the magnitude of the effect size. Also large GWAs on RA susceptibility had used fixed effect models [28]. In subanalyses a random effect model was also used.

For the analyses in Leiden-EAC, Groningen and Lund a multivariate normal regression model for longitudinal data was used with radiological score as response variable (see detailed descriptions elsewhere [29]). Adjustment variables were entered based on their association with joint destruction; in Leiden-EAC age, gender and the described treatment periods, in Groningen age and inclusion $\leq />1990$, as proxy for DMARD-therapy and in Lund adjustments were made for age.

In the Sheffield data-set, each patient had a set of hands and feet X-rays at one time-point. To make the scores comparable to the other data-sets, the estimated yearly progression rate was calculated, by dividing the total Larsen by the number of disease years at time of Xray [30]. The SNP association was tested in a linear regression analysis with log-transformed estimated yearly progression rate as outcome variable. No adjustments were applied as none of the tested variables was significantly associated with joint destruction.

In all data-sets, the radiological scores were logtransformed to obtain a normal distribution. Since the analyses were performed on the log-scale, the resulting coefficient on the original scale indicates how many fold the joint destruction increased per year of follow-up. Over a follow-up period of $n$ years the coefficient increases to the power of $n$.

Testing multiple SNPs on one data-sets leads to inflation of the p-value. It is debatable which multiple testing correction is best to use. In the current study the most conservative method was applied, the Bonferroni method, to reduce the chance on false-positive findings as much as possible. Since phase- 1 was used as identification phase, this correction was applied to the number of variants tested in phase-2.

\section{Haplotype analyses}

Haplotypes in OPG, RANK, RANKL and TRAF6 were studied. Haplotype blocks for the tag-SNPs were defined with Gabriel's method [31]. Haplotypes were assigned to each individual using PLINK 1.06 requiring a probability $>0.8$. Analyses of the haplotypes were performed with methods similar to those used for the analyses of the individual SNPs by now testing the presence of a haplotype compared to the absence of the haplotype.

\section{Results}

\section{Phase-1; SNP identification}

123 tagging SNPs in OPG $(\mathrm{n}=33), \operatorname{RANK}(\mathrm{n}=52)$, RANKL $(\mathrm{n}=21)$ and TRAF6 $(\mathrm{n}=17)$ were genotyped. Eleven SNPs were not analyzed because of a low typing success rates and three were out of HwE. From the 109 analyzed SNPs, 33 SNPs were significantly associated with joint destruction (see Additional file 1: Table S1); eighteen SNPs were located in $O P G$, ten in $R A N K$, four in RANKL and one in TRAF6. The associations of OPG SNPs were most prominent in the additive analyses. For RANK, RANKL and TRAF6 mainly recessive associations were observed (see Additional file 1: Table S1). The effect sizes observed represent the estimated relative progression rates per year. Consequently over a follow-up of a certain number of years, the effect sizes increases by the power of the number of follow-up years. For example, the estimate of 1.03 fold rate of joint destruction per year of the minor variant of OPG-1485305 (T) compared to patients with the common genotypes equals $1.23\left(1.03^{\wedge} 7\right)$ fold rate of joint destruction over 7 -years. In other words patients carrying one minor allele had over 7 -years a $23 \%$ higher rate of joint destruction (Figure 3).

Haplotype analyses were performed in addition to SNPs analyses. In phase-1, four haplotypes from one haplotype block in OPG were identified as possible more informative than the individual SNPs located in these haplotypes (see Additional file 2: Table S2) To tag these haplotypes in phase-2, one non-significant SNP of phase-1 was also typed in phase-2, rs1905785.

\section{Phase-2; meta-analysis}

Of the 33 significant SNPs from phase-1 19 were subsequently typed and analyzed in phase- 2 since they had a high enough minor allele frequency and their associations were independent of other SNPs (see Additional file 1: Table S1 and Additional file 3: Table S3 for detailed information). Therefore these nineteen SNPs plus 

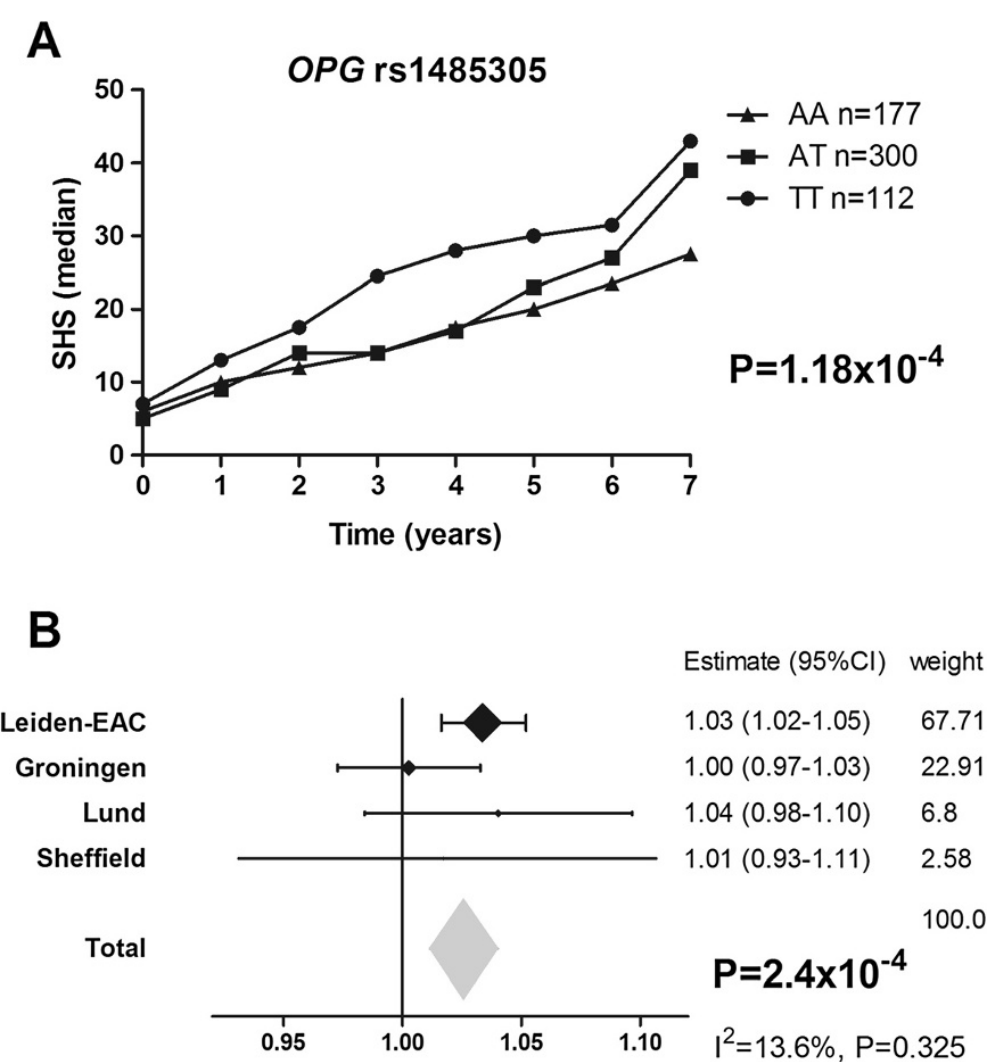

Figure 3 Depicted is OPG-rs1485305 in the Leiden-EAC (A) and in the meta-analysis on all data-sets (B). SHS = Sharp-van der Heijde score. The effect sizes are the estimated relative progression rates per year for the presence of the minor allele for OPG compared to patients without the minor allele. A) The presence of the minor variant of OPG-1485305 (T) is associated with a 1.03 fold rate of joint destruction per year compared to patients with the common genotypes in the Leiden-EAC. Since the effect sizes increases by the power of the number of follow-up years, these patients have a $1.23(1.03 \wedge 7)$ fold rate of joint destruction over 7-years, in other words a $23 \%$ higher rate of joint destruction. B) The meta-analysis is based on a fixed effect model, which is applied to genetic studies to test whether there is statistically significant effect; generalizability of the effect is of less importance. Consequently, this method is less suitable to estimate the effect size overall. Therefore, the estimated effect of the meta-analysis is depicted in gray. The $I^{2}$ was $13.6 \%$ and the $p$-value for heterogeneity was 0.325 . The $p$-value for a random model was 0.004 and the effect size 1.23 (see also Additional file 3: Table S3).

one SNP to allow haplotype analysis were studied in phase-2. As expected due to insufficient power of the replication cohorts, the $95 \%$ confidence intervals in each of the three cohorts separately all included 1 (see Additional file 3: Table S3). The SNPs were subsequently analyzed in all 1,418 patients in an inverse variance weighting meta-analysis. Here, ten SNPs (six located in $O P G$ and four in $R A N K$ ) were significantly associated with rate of joint destruction $(0.04>P>$ $2.4 \times 10^{-4}$ ). After correction for testing 20 SNPs using the Bonferroni method, one SNP was significantly associated with the rate of joint destruction; OPG-r1485305 (Figure 3). Patients carrying at least one minor allele of OPG-rs1485305 (T) had a higher rate of joint destruction as compared to patients without this minor allele $\left(P=2.35 \times 10^{-4}\right.$, uncorrected $P$-value $)$.

When the association of OPG-rs1485305 with the rate of joint destruction was studied in ACPA-negative and ACPA-positive patients separately in the Leiden-EAC, rs1485305 was significantly associated with progression of joint destruction in ACPA-negative patients (1.29 95\% CI 1.10-1.50, $P=0.001$ ) but not in ACPA-positive patients although a similar trend was observed (1.14 95\% CI 0.97$1.34, P=0.11)$. Also when the analysis in the total group of RA -patients was adjusted for ACPA and rheumatoid factor the association remained significant $(1.2095 \% \mathrm{CI}$ 1.07-1.35, $P=0.02$ ). Similarly, adjusting for the level of inflammation measured by $\mathrm{C}$-reactive protein levels at baseline) did not affect the effect size and significance was maintained (1.29 95\% CI 1.14-1.46, $P=0.0005)$. This suggests that the association between OPG-rs1485305 and radiological progression in the Leiden data was not confounded by auto-antibodies or CRP-levels. Unfortunately, our data on these adjustment factors were too limited to confirm this with the other cohorts.

In phase-2, none of the tested haplotypes provided additional information to the results of the individual SNPs (see Additional file 2: Table S2). 


\section{Discussion and conclusion}

The variance in joint destruction between RA-patients is considerable and the mechanisms driving these differences are thus far scarcely understood. Part of the severity of joint damage is explained by the cumulative levels of inflammation [32] though this explanation is incomplete. We reasoned that individual susceptibility of bones to erode may affect the severity of joint destruction in RA as well. We therefore studied the association of genetic variants in OPG, RANK, RANKL and TRAF6 with joint destruction as the products of these genes together constitute a pathway that is crucial in osteoclastogenesis and bone resorption. One SNP, OPG-rs14085305, was observed to significantly associate with progression of joint destruction in RA.

The association between OPG-rs14085305 with progression of joint destruction in RA was independent of autoantibody positivity. However, after stratification for ACPA the association was only significant in the ACPA-negative group. This seemingly counterintuitive result could be explained by a strong effect of the association in the ACPA-negative group, by which the multivariable analysis remained significant. Furthermore, since the effect sizes iwere quite similar in the ACPA-positive and ACPAnegative subgroups, it is also possible that the association in ACPA-positive patients was insufficiently powered to obtain statistical significance in this subgroup. Larger datasets of ACPA + RA-patients would be required to further elucidate the association between rs14085305 and joint damage progression in ACPA + RA.

OPG is expressed in several cells and tissues among which osteoblasts, chondrocytes and bone marrow. Serum OPG is decreased in the synovium and serum of RApatients [33]. Low serum OPG/RANKL ratio's have been associated with progression of joint destruction [12]. In addition, low OPG/RANKL ratio's in cartilage are associated with the degree of joint destruction is situated in the 5-UTR flanking region of OPG. Thus far no functional data on this variant exist, hence the mechanism by which rs1485305 affects OPG expression or function is yet unknown. Nonetheless, OPG-rs1485305 was recently also observed to associate with bone mineral density loss, which strengthens the relevance of this SNP in relation to bone and joint disease [34].

Our group recently performed a genome-wide study on joint damage progression in ACPA-positive RA-patients [35] and evaluated the genetic variants included on the Immunochip in relation to joint damage progression in RA [36]. In these studies the current variant OPG-rs1485305 was not identified as a risk factor for joint damage progression as this variant was not (also no proxies with $\mathrm{r}^{2}>0.80$ ) included in both these genotyping platforms.

An advantage of the four studied data-sets is that the evaluated RA-patients were treated in an era when treatment was not as aggressive as nowadays. Hence, the radiologic progression rates of the studied patients are more reflective of the natural course of RA than that of recently treated patients. Some data-sets included patients from different periods that had received different treatment regiments potentially affecting an association with progression of joint destruction. Since treatment may be an effect modifier masking associations with radiological progression, the analyses in these data-sets were adjusted for inclusion period as proxy for treatment.

Replication data-sets are ideally larger than the initial data-set, since effects sizes are generally smaller at a replication stage. A limitation of the present study is that we were not able to include replication data-sets that contained more X-rays than the initial data-set and of which the RA-patients were "conventionally" treated. Most likely, few of such longitudinal data-sets exist. In one data-set only one radiograph per patient was available; it is known that this results in less precise estimations of the progression rate compared to having serial radiological measurements [37]. This was here also depicted by a broader confidence interval of the effect estimate of the Sheffield data. Nonetheless the results of a meta-analysis on rs1485305 without the Sheffield data was still significant ( $\mathrm{p}=0.0024$ data not shown).

Since the number of patients and the number X-rays of each data-set separate were insufficient to allow well powered analyses, the data of the different replication cohorts were summarized in inverse variance weighting metaanalyses. Notably, also the replication data-sets combined contained less radiological measurements than in phase-1. Importantly, the effects of OPG-rs1485305 went into the same direction in each dataset supporting the validity of the results. In addition, the heterogeneity $\left(I^{2}\right)$ of the data for OPG-rs1485305 was only $13.6 \%$, making it less likely that OPG-rs1485305 is a false positive finding due to differences between the four cohorts. This $I^{2}$ supports the use of a fixed effects meta-analysis, but also when a random effect model was used OPG-rs1485305 remained significant (Additional file 3: Table S3).

To further prevent false positive findings due to performing multiple comparisons, data were corrected for multiple testing using the Bonferroni method. This was done in phase-2, since phase- 1 was used as discovery phase. However, the association of OPG-rs1485305 with the rate of joint destruction would also have remained significant when Bonferroni correction would have been applied in phase-1 correcting for 109 SNPs. This further consolidates the validity on the results on OPG-rs1485305.

Interestingly, several other studies, among which genome-wide association studies, have revealed several genetic variants in RANKL/RANK/OPG to associate with bone mineral density or osteoporosis. Therefore during the tagging and SNP selection phase, such variants were forced into the selection. Interestingly (except for 
rs1485305) none of these variants were significantly associated with progression of joint destruction in phase-2. Conceptually, the balance between osteoblast and osteoclast activity is crucial both in osteoporosis and joint destruction in RA but the individual genetic variants predisposing to such systemic or local bone loss are largely dissimilar.

During the SNP selection phase, three coding SNPs were also prioritized; these three were also not significantly associated after correction for multiple testing in phase-2. A coding variant in RANK, rs8092336, was significantly associated with joint destruction in phase-1 and phase-2, but did not remain significant after correction for 20 tests. The same was observed for a coding SNP in $O P G$, rs2073618, which has also been associated with bone mineral density [15]. These two SNPs could potentially be associated with progression of joint destruction, but the chance of a Type-I error is too large to conclude this on the basis of current data.

Finally, TRAF6 was chosen as candidate gene because it is a relevant signal transducer in the Nuclear Factor $\mathrm{\kappa} B$ pathway. Rs540386 in TRAF6 was previously also identified as a risk locus for RA susceptibility, though TRAF6 was not genome-wide significant in a recent study on more than 11,000 cases and 15,000 controls [29,38]. Our candidate gene study had started a year before the first report of TRAF6 and RA susceptibility was published, hence this report had not affected the choice of TRAF6 as candidate gene. The susceptibility SNP rs540386 is in close LD $\left(r^{2}=0.94\right)$ with one of the tag SNP evaluated in the current study (rs11033647). This SNP was not associated with the rate of joint destruction in our analyses. Although this could be a false-negative finding, it is also possible that different genetic variants are involved in RAsusceptibility and the progression of joint destruction.

The current study included patients only. Whether the rs1485305 is also relevant for RA-susceptibility was not studied here. Nonetheless, several genome-wide studies have been performed on RA-susceptibility and $>40$ genetic susceptibility factors have been identified but rs1485305 in OPG was not one of them. Presumably different genetic factors are involved in developing RA and in progression of structural damage.

In conclusion, with a candidate gene approach evaluating patients of four different cohorts, we found association of a genetic variant in $O P G$ with an increased rate of joint destruction in RA. The present data support the role of OPG in joint destruction in RA by indicating that the risk allele of rs1485305 may affect the homeostasis in bone.

\section{Additional files}

Additional file 1: Table S1. Results of SNP analysis phase-1 in the Leiden-EAC.
Additional file 2: Table S2. Haplotype analyses.

Additional file 3: Table S3. Results of the significant SNPS of the phase-l, phase-II and the meta-analysis (fixed as well as random effects) The fixed effect model was our main model of choice, guided by the argument of Lebrec et al. [27] that the heterogeneity is of less importance in genetic studies when the search for a significant association in the first studies is of more importance than the magnitude of the effect size. To provide insight into the heterogeneity of the cohorts the 12 for heterogeneity was calculated. In this table also the results of a random meta-analysis is provided. The final conclusion that

OPG-rs1485305 is significantly associated with rate of joint destruction is irrespective of which model for meta-analysis is used.

\section{Abbreviations}

DMARDs: Disease modifying anti-rheumatic drugs; EAC: Early Arthritis Clinic; HwE: Hardy-Weinberg equilibrium; ICC: Intra-correlation coefficient; MAF: Minor allele frequency; NF: Nuclear Factor; NSAIDs: Non-steroid anti-inflammatory drugs; OPG: Osteoprotegerin; RA: Rheumatoid Arthritis; RANK: Receptor Activator for Nuclear Factor k B; RANKL: Receptor Activator for Nuclear Factor K B Ligand; SD: Standard deviation; SHS: Sharp-van der Heijde; SNP: Single nucleotide polymorphism; TRAF6: Tumour necrosis Receptor Associated Factor-6.

\section{Competing interests}

None of the authors has any financial conflicts of interest to declare.

\section{Authors' contributions}

RK: design, collection, analyses, manuscript (re)writing, final approval. $\mathrm{AH}$ : design, collection, manuscript writing, final approval. ND: data collection, analyses, manuscript rewriting, final approval. GW: data collection,

manuscript rewriting, final approval. EL: data collection, manuscript rewriting final approval. ML: data collection, manuscript rewriting, final approval. BK: data collection, manuscript rewriting, final approval. RT: data collection, manuscript rewriting, final approval. TS: data collection, manuscript rewriting, final approval. TH: data collection, manuscript rewriting, final approval. EB: data collection, manuscript rewriting, final approval. DR analyses, manuscript rewriting, final approval. JS: analyses, data collection, manuscript rewriting, final approval. JH data analysis, manuscript rewriting, conception final approval. All authors read and approved the final manuscript.

\section{Acknowledgements}

The work of A.H.M. van der Helm-van Mil is supported by The Netherlands Organization for Health Research and Development. The work of R. Knevel is supported by the Dutch Arthritis Association. The research has been funded by The European Community Seventh Framework Program FP7 Health-F22008-223404 (Masterswitch), the IMI JU funded project BeTheCure, contract no 115142-2. We thank all the RA-patients who participated in this study.

\section{Author details}

${ }^{1}$ Department of Rheumatology, Leiden University Medical Center, Leiden, the Netherlands. ${ }^{2}$ Department of Rheumatology, Lund University, Lund, Sweden. ${ }^{3}$ Department of Rheumatology and Clinical Immunology, University of Groningen, University Medical Center Groningen, Groningen, the Netherlands. ${ }^{4}$ Complex Genetics Section, Department of Medical Genetics, University Medical Centre Utrecht, Utrecht, the Netherlands. ${ }^{5}$ Department of Medical Statistics, Leiden University Medical Center, Leiden, the Netherlands. ${ }^{6}$ School of Medicine and Biomedical Sciences, The University of Sheffield, Sheffield, UK.

Received: 9 November 2013 Accepted: 15 April 2014

Published: 7 May 2014

\section{References}

1. van der Heijde DM, van Riel PL, van Leeuwen MA, van 't Hof MA, van Rijswijk MH, van de Putte LB: Prognostic factors for radiographic damage and physical disability in early rheumatoid arthritis. A prospective follow-up study of 147 patients. Br J Rheumatol 1992, 31:519-525.

2. de Rooy DP, van der Linden MP, Knevel R, Huizinga TW, van der Helm-van Mil AH: Predicting arthritis outcomes-what can be learned from the Leiden Early Arthritis Clinic? Rheumatology (Oxford) 2011, 50:93-100. 
3. van der Helm-van Mil AH, Kern M, Gregersen PK, Huizinga TW: Variation in radiologic joint destruction in rheumatoid arthritis differs between monozygotic and dizygotic twins and pairs of unrelated patients. Arthritis Rheum 2006, 54:2028-2030.

4. Knevel R, Gröndal G, Huizinga TW, Visser AW, Jónsson H, Víkingsson A, Geirsson AJ, Steinsson K, van der Helm-van Mil AH: Genetic predisposition of the severity of joint destruction in rheumatoid arthritis: a population-based study. Ann Rheum Dis 2012, 71:707-709.

5. Kong YY, Feige U, Sarosi I, Bolon B, Tafuri A, Morony S, Capparelli C, Li J, Elliott R, McCabe S, Wong T, Campagnuolo G, Moran E, Bogoch ER, Van G, Nguyen LT, Ohashi PS, Lacey DL, Fish E, Boyle WJ, Penninger JM: Activated $T$ cells regulate bone loss and joint destruction in adjuvant arthritis through osteoprotegerin ligand. Nature 1999, 402:304-309.

6. Gravallese EM, Galson DL, Goldring SR, Auron PE: The role of TNF-receptor family members and other TRAF-dependent receptors in bone resorption. Arthritis Res 2001, 3:6-12.

7. Bromley M, Woolley DE: Chondroclasts and osteoclasts at subchondral sites of erosion in the rheumatoid joint. Arthritis Rheum 1984, 27:968-975.

8. Leisen JCC, Duncan H, Riddle JM, Pitchford WC: The erosive front: a topographic study of the junction between the pannus and the subchondral plate in the macerated rheumatoid metacarpal head. J Rheumatol 1988, 15:17-22.

9. Horwood NJ, Kartsogiannis V, Quinn JMW, Romas E, Martin TJ, Gillespie MT: Activated T lymphocytes support osteoclast formation in vitro. Biochem Biophys Res Commun 1999, 265:144-150.

10. Takayanagi H, lizuka H, Juji T, Nakagawa T, Yamamoto A, Miyazaki T, Koshihara Y, Oda H, Nakamura K, Tanaka S: Involvement of recepto activator of nuclear factor kappa-B ligand/osteoclast differentiation factor in osteoclastogenesis from synoviocytes in rheumatoid arthritis. Arthritis Rheum 2000, 43:259-269.

11. Martínez-Calatrava MJ, Prieto-Potín I, Roman-Blas JA, Tardio L, Largo R, Herrero-Beaumont G: RANKL synthesized by articular chondrocytes contributes to juxta-articular bone loss in chronic arthritis. Arthritis Res Ther 2012, 14:R149.

12. Geusens PP, Landewé RB, Garnero P, Chen D, Dunstan CR, Lems WF, Stinissen $P$, van der Heijde DM, van der Linden S, Boers M: The ratio of circulating osteoprotegerin to RANKL in early rheumatoid arthritis predicts later joint destruction. Arthritis Rheum 2006, 54:1772-1777.

13. Styrkarsdottir U, Halldorsson BV, Gretarsdottir S, Gudbjartsson DF, Walters GB, Ingvarsson T, Jonsdottir T, Saemundsdottir J, Center JR, Nguyen TV, Bagger Y, Gulcher JR, Eisman JA, Christiansen C, Sigurdsson G, Kong A, Thorsteinsdottir U, Stefansson K: Multiple genetic loci for bone mineral density and fractures. N Engl J Med 2008, 358:2355-2365.

14. Richards JB, Rivadeneira F, Inouye M, Pastinen TM, Soranzo N, Wilson SG, Andrew T, Falchi M, Gwilliam R, Ahmadi KR, Valdes AM, Arp P, Whittaker P, Verlaan DJ, Jhamai M, Kumanduri V, Moorhouse M, van Meurs JB, Hofman A, Pols HA, Hart D, Zhai G, Kato BS, Mullin BH, Zhang F, Deloukas P, Uitterlinden AG, Spector TD: Bone mineral density, osteoporosis, and osteoporotic fractures: a genome-wide association study. Lancet 2008 371:1505-1512.

15. Hsu YH, Niu T, Terwedow HA, Xu X, Feng Y, Li Z, Brain JD, Rosen CJ, Laird N, $\mathrm{Xu} X$ : Variation in genes involved in the RANKL/RANK/OPG bone remodeling pathway are associated with bone mineral density at different skeletal sites in men. Hum Genet 2006, 118:568-577.

16. Rivadeneira F, Styrkársdottir U, Estrada K, Halldórsson BV, Hsu YH, Richards JB, Zillikens MC, Kavvoura FK, Amin N, Aulchenko YS, Cupples LA, Deloukas P, Demissie S, Grundberg E, Hofman A, Kong A, Karasik D, van Meurs JB, Oostra B, Pastinen T, Pols HA, Sigurdsson G, Soranzo N, Thorleifsson G, Thorsteinsdottir U, Williams FM, Wilson SG, Zhou Y, Ralston SH, van Duijn $C M$, et al: Twenty bone-mineral-density loci identified by large-scale meta-analysis of genome-wide association studies. Nat Genet 2009, 41:1199-1206.

17. van der Heijde D: How to read radiographs according to the Sharp/van der Heijde method. J Rheumatol 2000, 27:261-263.

18. Rau R, Herborn G: A modified version of Larsen's scoring method to assess radiologic changes in rheumatoid arthritis. J Rheumatol 1995 22:1976-1982

19. Mewar D, Coote A, Moore DJ, Marinou I, Keyworth J, Dickson MC, Montgomery DS, Binks MH, Wilson AG: Independent associations of anti-cyclic citrullinated peptide antibodies and rheumatoid factor with radiographic severity of rheumatoid arthritis. Arthritis Res Ther 2006, 8:R128.
20. Fex E, Jonsson K, Johnson U, Eberhardt K: Development of radiographic damage during the first 5-6 yr of rheumatoid arthritis. A prospective follow-up study of a Swedish cohort. British. J Rheumatol 1996, 35:1106-1115.

21. Larsen A, Dale K, Eek M: Radiographic evaluation of rheumatoid arthritis and related conditions by standard reference films. Acta Radiol Diagn (Stockh) 1977, 18:481-91.

22. Lindqvist $E$, Jonsson K, Saxne T, Eberhardt K: Course of radiographic damage over 10 years in a cohort with early rheumatoid arthritis. Ann Rheum Dis 2003, 62:611-616.

23. Barrett JC, Fry B, Maller J, Daly MJ: Haploview: analysis and visualization of LD and haplotype maps. Bioinformatics 2005, 21:263-265.

24. Dubois PC, Trynka G, Franke L, Hunt KA, Romanos J, Curtotti A, Zhernakova A, Heap GA, Adány R, Aromaa A, Bardella MT, van den Berg LH, Bockett NA, de la Concha EG, Dema B, Fehrmann RS, Fernández-Arquero M, Fiatal S, Grandone E, Green PM, Groen HJ, Gwilliam R, Houwen RH, Hunt SE, Kaukinen K, Kelleher D, Korponay-Szabo I, Kurppa K, MacMathuna P, Mäki M, et al: Multiple common variants for celiac disease influencing immune gene expression. Nat Genet 2010, 42:295-302.

25. van der Helm-van Mil AH, Knevel R, van der Heijde D, Huizinga TW: How to avoid phenotypic misclassification in using joint destruction as an outcome measure for Rheumatoid Arthritis? - Sensitive measuring of joint destruction in RA. Rheumatology (Oxford) 2010, 49:1429-1435.

26. Lassere M: Pooled metaanalysis of radiographic progression: comparison of Sharp and Larsen methods. J Rheumatol 2000, 27:269-275.

27. Lebrec JJ, Stijnen T, van Houwelingen HC: Dealing with heterogeneity between cohorts in genomewide SNP association studies. Stat Appl Genet Mol Biol 2010, 9:Article 8

28. Knevel R, Krabben A, Brouwer E, Posthumus MD, Wilson AG, Lindqvist $E$, Saxne T, de Rooy D, Daha N, van der Linden MP, Stoeken G, van Toorn L, Koeleman B, Tsonaka R, Zhernakoza A, Houwing-Duistermaat JJ, Toes R, Huizinga TW, van der Helm-van MA: Genetic variants in IL-15 Associate with Progression of Joint Destruction in Rheumatoid Arthritis, a Multi Cohort Study. Ann Rheum Dis 2012, 71:1651-1657.

29. Eyre S, Bowes J, Diogo D, Lee A, Barton A, Martin P, Zhernakova A, Stahl E, Viatte S, McAllister K, Amos Cl, Padyukov L, Toes RE, Huizinga TW, Wijmenga C, Trynka G, Franke L, Westra HJ, Alfredsson L, Hu X, Sandor C, de Bakker PI, Davila S, Khor CC, Heng KK, Andrews R, Edkins S, Hunt SE, Langford C, Symmons $D$, et al: High density genetic mapping identifies new susceptibility loci for rheumatoid arthritis. Nat Genet 2012, 44:1336-1340

30. Strand V, Landéwé $R$, van der Heijde $D$ : Using estimated yearly progression rates to compare radiographic data across recent randomised controlled trials in rheumatoid arthritis. Ann Rheum Dis 2002, 61:ii64-ii66.

31. Gabriel SB, Schaffner SF, Nguyen H, Moore JM, Roy J, Blumenstiel B, Higgins J, DeFelice M, Lochner A, Faggart M, Liu-Cordero SN, Rotimi C, Adeyemo A, Cooper R, Ward R, Lander ES, Daly MJ, Altshuler D: The structure of haplotype blocks in the human genome. Science 2002, 21:2225-2229.

32. Knevel R, van Nies JA, le Cessie S, Huizinga TW, Brouwer E, van der Helmvan Mil AH: Evaluation of the contribution of cumulative levels of inflammation to the variance in joint destruction in rheumatoid arthritis. Ann Rheum Dis 2013, 72:307-308.

33. Skoumal M, Kolarz G, Haberhauer G, Woloszczuk W, Hawa G, Klingler A: Osteoprotegerin and the receptor activator of NF-kappa B ligand in the serum and synovial fluid. A comparison of patients with longstanding rheumatoid arthritis and osteoarthritis. Rheumatol Int 2005, 26:63-69.

34. Richards JB, Kavvoura FK, Rivadeneira F, Styrkársdóttir U, Estrada K, Halldórsson BV, Hsu YH, Zillikens MC, Wilson SG, Mullin BH, Amin N, Aulchenko YS, Cupples LA, Deloukas P, Demissie S, Hofman A, Kong A, Karasik D, van Meurs JB, Oostra BA, Pols HA, Sigurdsson G, Thorsteinsdottir U, Soranzo N, Williams FM, Zhou Y, Ralston SH, Thorleifsson G, van Duijn CM, Kiel DP, et al: Collaborative Meta-analysis: Associations of 150 Candidate Genes With Annals of Internal Medicine Osteoporosis and Osteoporotic Fracture. Ann Intern Med 2009, 20:151. 528-537.

35. Knevel R, Klein K, Somers K, Ospelt C, Houwing-Duistermaat JJ, van Nies JA, de Rooy DP, de Bock L, Kurreeman FA, Schonkeren J, Stoeken-Rijsbergen G, Helmer Q, van der Linden MP, Kern M, Manjarrez-Orduno N, RodriguezRodriquez L, Stinissen P, Huizinga TW, Toes RE, Gay S, Gregersen PK, Somers $\checkmark$, van der Helm-van Mil AH: Identification of a genetic variant for joint damage progression in autoantibody-positive rheumatoid arthritis. Ann Rheum Dis 2013, 16 [Epub ahead of print].

36. Knevel R, de Rooy DP, Zhernakova A, Gröndal G, Krabben A, Steinsson K, Wijmenga C, Cavet G, Toes RE, Huizinga TW, Gregersen PK, van der Helm-van 
Mil AH: Association of variants in IL2RA with progression of joint destruction in rheumatoid arthritis. Arthritis Rheum 2013, 65:1684-1693.

37. Knevel R, Tsonaka R, le Cessie S, van der Linden MP, Huizinga TW, van der Heijde DM, Houwing-Duistermaat J, van der Helm-van Mil AH: Comparison of methodologies for analysing the progression of joint destruction in rheumatoid arthritis. Scand J Rheumatol 2013, 42:182-189.

38. Raychaudhuri S, Thomson BP, Remmers EF, Eyre S, Hinks A, Guiducci C, Catanese JJ, Xie G, Stahl EA, Chen R, Alfredsson L, Amos Cl, Ardlie KG,

Consortium BIRAC, Barton A, Bowes J, Burtt NP, Chang M, Coblyn J, Costenbader KH, Criswell LA, Crusius JB, Cui J, De Jager PL, Ding B, Emery P, Flynn E, Harrison P, Hocking LJ, Huizinga TW, et al: Genetic variants at $\mathrm{CD} 28$, PRDM1 and CD2/CD58 are associated with rheumatoid arthritis risk. Nat Genet 2009, 41:1313-1318.

\section{doi:10.1186/ar4558}

Cite this article as: Knevel et al: A genetic variant in osteoprotegerin is associated with progression of joint destruction in rheumatoid arthritis. Arthritis Research \& Therapy 2014 16:R108.

\section{Submit your next manuscript to BioMed Central and take full advantage of:}

- Convenient online submission

- Thorough peer review

- No space constraints or color figure charges

- Immediate publication on acceptance

- Inclusion in PubMed, CAS, Scopus and Google Scholar

- Research which is freely available for redistribution 